\title{
Deploying PaaS for Accelerating Cloud Uptake in the Guifi Community Network
}

\author{
Javi Jiménez, Pau Escrich, Roger Baig \\ Fundació Privada per a la Xarxa Lliure, \\ Oberta i Neural guifi.net \\ Mas l'Esperana, 08503 Gurb, Catalonia \\ \{javi.jimenez, roger.baig, pau.escrich\}@ guifi.net
}

\author{
Felix Freitag, Leandro Navarro \\ Department of Computer Architecture \\ Universitat Politècnica de Catalunya \\ Barcelona, Spain \\ $\{$ felix, leandro\}@ac.upc.edu
}

\begin{abstract}
Community networks is an emerging model in which communities of citizens build and own open shared networks. But currently, cloud computing infrastructures, common in today's Internet, hardy exist in community networks. We present in this paper our approach for bringing clouds into community networks. The main element of our strategy for achieving cloud uptake is the deployment of the Guifi-Community-Distribution (GCODIS) on all cloud nodes, a distribution containing common services and applications. We argue in the paper the reasons why this approach is appropriate for the scenario of community networks, where the user acceptance needs to be gained. We show the steps and the on-going process with which we actually implement our approach in the Guifi community network. With these key elements in place, we conclude that we are closer to the vision that the users of community networks ultimately will not need to consume cloud applications from the Internet, but find them within the community network. An interesting open issue is to what extend our approach can be applied to the more generic volunteer computing scenario.
\end{abstract}

Index Terms-community networks; cloud computing;

\section{INTRODUCTION}

Community networking, also known as bottom-up networking, is an emerging model for the Future Internet, where local communities of citizens build, operate and own open IP-based networks. These networks are usually run by non-profit organizations with the support from local public authorities and private sector organisations, cooperating under an open model with local stakeholders and commercial service providers to develop community services, including local networking, voice connections and Internet access.

Hundreds of community networks operate across the globe, in rural and urban, rich and poor areas. Networks exist almost everywhere e.g. in the USA, WasabiNet is running in St. Louis, Missouri while The Personal Telco Project is deployed in Portland, Oregon. In Latin America, numerous networks exist and cooperate, e.g. Bogota Mesh and Monte Video Libre. For instance in Melbourne, Australia, Melbourne Wireless is a quickly growing community network. In Europe, FunkFeuer in Austria, AWMN in Athens, Greece, Guifi.net in Spain and Freifunk in Germany have reached thousands of nodes.

While community networks are a successful case of network sharing among a collective, computing and storage resource sharing, which is now common practice in today's Internet through cloud computing, hardly exists in community networks. Community clouds[1] are a vision that extend the collective action of community networks to the sharing of all computing resources: A cloud hosted on community-owned computing and communication resources, managed by the community, providing to the community services of local interest.

Since community clouds are built upon community networks, they inherit many of the features of community networks[2][3]. Therefore, such a community cloud is expected to have the following properties:

- Autonomy: Individual cloud systems are set up and managed independently by different owners.

- Security: Though there are many security challenges[4], leverage on choosing applications that include security in their design.

- Self-Management: Self-management of nodes needs to be pursued to continue providing services without disruption.

- Usefulness: A set of distributed applications needs to be deployed on the shared cloud infrastructure that are valuable for the community.

- Ease of Use: Clouds administration capabilities need to be low since most users of the community cloud will not be proficient in cloud technologies.

- Incentives for contribution: Users will add nodes, offer resources and get other users' resources. Cloud service therefore should reward the users that make contributions, where contributions can be, among others, hardware, knowledge, developments, support, node administration.

Figure 1 illustrates typical nodes of a community network, like community nodes with a router and some server or clients attached to it. In addition, however, these community nodes have cloud resources attached to them. With this hardware the community cloud is built.

We argue that sharing in community networks should go beyond bandwidth. Sharing should be extended to other computing resources through clouds, to offer in a flexible way more applications inside of the community network, to boost the potential of such open, neutral and collectively managed communication infrastructures. In the following sections we 


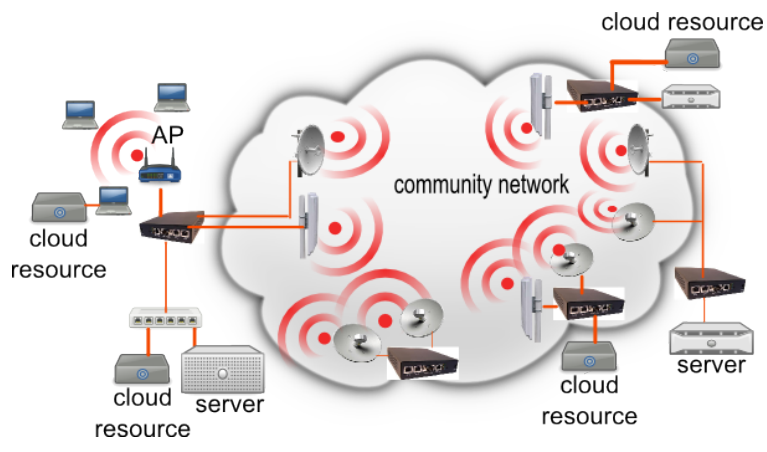

Fig. 1. Nodes in a community network with cloud resources

present our approach in which PaaS plays a central role.

\section{Key Elements to bRing Cloud into Community NETWORKS}

We discuss in this section the key elements we have identified as the base for the success of clouds in community networks.

1) Focus on cloud applications and services: For a sustainable community cloud, users must find interesting services provided by the cloud. A key decision of our approach is not to focus on building a customized infrastructure service, but put emphasis on building a customized Linux distribution which contains platform and application services pre-installed. Only with the availability of attractive applications and services in the cloud, more community users will join. As a consequence, infrastructure services (e.g. virtual machine management) in our approach can be performed by any cloud management platform, e.g. OpenStack, OpenNebula, Eucalyptus, Proxmox.

2) Integration of the cloud in the community network management software: For configuration, cloud hosts can be selected by the user in the community network web interface simply as devices to be attached to community network super nodes, similar to other devices such as routers, bridges etc. The support of the community network web for cloud devices is an important demonstration to the community network end user that cloud devices are supported in the community network.

3) Access to community network nodes for initial cloud deployment: Our approach is to deploy an operational prototype of the community cloud, which is needed such that the end user of community networks can really test and use cloud services. If our cloud would have been provided as a laboratory experiment, it would hardly ever become integrated in the community network.

4) Sustainability of the community cloud: Community networks are maintained by volunteers. While the building and maintenance of the network layer is already complex, the administration of a cloud might require even more specific knowledge. In order make the cloud administration sustainable, open source software that is in production stage and which has a wide user community must be applied. But still, in order to achieve an ecosystem of cloud providers and consumers, incentives for the users such as quality of experience should be integrated in the cloud system.

\section{A Case Study: Deployment of the Community CLOUd IN GUIFI.NET COMMUNITY NETWORK}

In this section we describe how in the currently on-going real cloud deployment in the Guifi.net community network these previously identified elements appear and how they are brought into practice.

\section{A. Guifi-Community-Distro and Cloud services}

The Guifi-Community-Distro (GCODIS) is the operating system image which we have prepared to be placed in the virtual machines of each cloud node. It is a Debian-based distribution which has been equipped with a set of basic platform services and applications. Some of these services are common with other Guifi devices, such as the graphics server and the proxy service. Other services, such as Avahi ${ }^{1}$, Tahoelafs $^{2}$ and owncloud ${ }^{3}$ have been explicitly added to the GuifiCommunity-Distro as cloud platform and application services. Therefore, this solution of providing the Guifi-CommunityDistro addresses the first key element explained in the previous section.

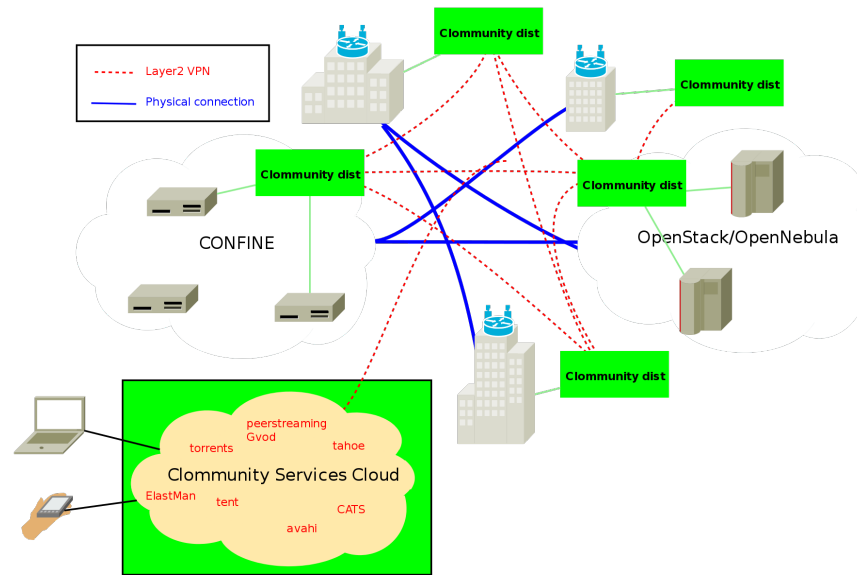

Fig. 2. Global community cloud deployment scenario

Figure 2 indicates some of the already offered and planned services. The Avahi Zeroconf platform service is particularly important for the cloud system since it allows the publication and location of services, and with additional software even outside of the LAN (though further research and additional tests are needed). This way services can be seen from any node from inside and outside of the cloud, and with the use of the browse capabilities of the Avahi Zeroconf implementation, the cloud user experiences a vision of an uniform integration of services within the cloud.

Figure 3 shows a layered architectural view that we use which integrates the users, software, hardware and the network. From a top-down perspective, the Guifi-CommunityDistro is our solution for the upper three layers: This is providing pre-installed in a basic Linux Debian as software

\footnotetext{
${ }^{1}$ http://avahi.org/

${ }^{2}$ https://tahoe-lafs.org/ warner/pycon-tahoe.html

${ }^{3}$ http://owncloud.org/
} 
system, additional services and applications, like the cloud internal Ahavi service and but also other services which reach the end users, such as owncloud and Tahoe-Lafs for distributed storage.

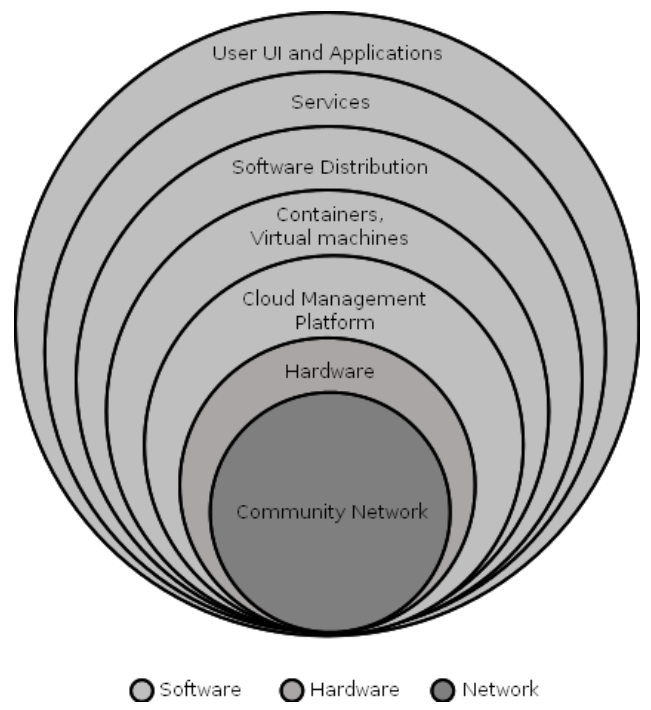

Fig. 3. Network and service architecture

\section{B. Steps to install the hardware of a community cloud node}

In order to install a community cloud node, the Guifi user applies the same mechanism he/she already knows when deploying a normal nodes inside the Guifi community network. Once a node is placed in the Guifi web, the final step is to add the cloud resource, as shown in Figure 4: It can be seen that the web interface allows the user to select the Confine/Clommunity device, which is the cloud resource. This solution implements the second key element of the previous section.

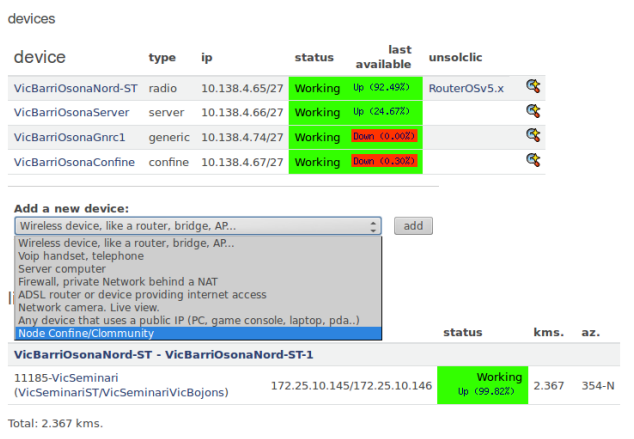

Fig. 4. Adding a cloud resource to the Guifi community network node (source: http://guifi.net/en/node/1655)

\section{Cloud management platform}

The cloud hosts need to be managed, in terms of their virtual machines, for monitoring, update etc. Currently, we use the management software of the Community-Lab testbed ${ }^{4}$ devel- $^{-}$ oped by the CONFINE project[5], with which the community

\footnotetext{
${ }^{4}$ http://community-lab.net
}

boxes (see next section) are integrated into its management services. Different to other open source cloud management platforms, the management software of the Community-Lab allows us manage cloud resources with OpenWRT as host operating system, which is the case of the community boxes. Similar to other cloud management platform, as illustrated in Figure 5, the user can select the Guifi-Community-Distro to be loaded as operating system image into the virtual machines of a slice. Our access through Community-Lab to make real deployments in the Guifi community networks is the way to fulfill the third key element.

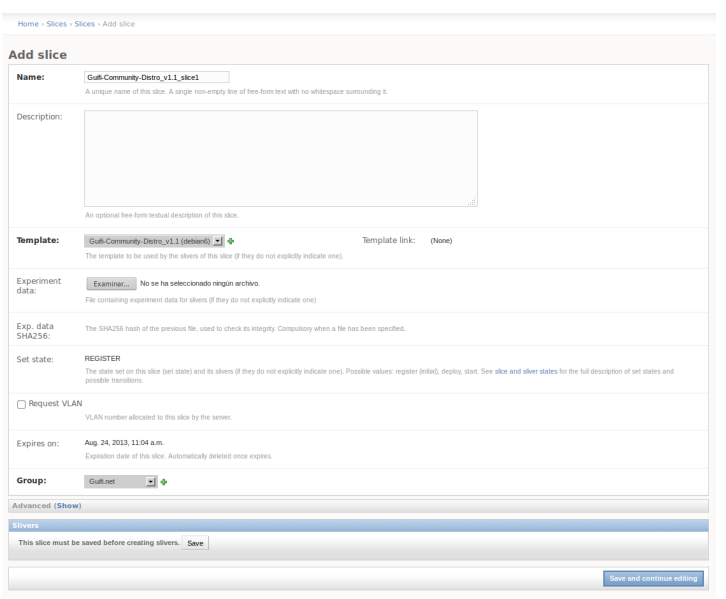

Fig. 5. Create at virtual machine with the Guifi-Community-Distro template

\section{Community Box as cloud hosts}

The hardware that we currently deploy as hosts of the community cloud are bare bones devices of type Jetway JBC362F36 (Figure 6), which we call Community Box (CB). These devices were chosen due to their low power consumption in order to be operational at the user premises at low cost in 24/7 mode and due to being fanless and without moving parts, with a zero level of noise, providing low levels of environmental contamination. The user as cloud contributer,however, can choose to donate other kind of hardware for the cloud nodes, such as other micro-devices, other barebone devices or even high end servers. With this flexibility regarding hardware contributions, we enable a fast uptake of the community cloud, necessary to reach sustainable operation. Once a critical mass of cloud infrastructure is reached, however, a limited catalogue of devices might be suggested to new participants, in order to be able to produce a limited set of targeted guides for maintanance.

\section{RELATED WORK}

The vision of community cloud computing has been described in [1]. The authors outline the conditions of a community and how a cloud system could be designed for such conditions. While the theoretical contribution of this paper is very relevant for our work, the authors of the cited paper did not really develop any real cloud system beyond their proposed architecture. 


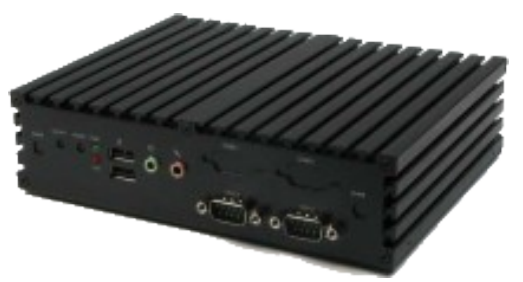

Fig. 6. Community Box as host in the community cloud

On the level of complete systems for community cloud computing, there are a few research prototypes, but none for community networks such as targeted by us. Skadsem et al. [6] provide applications for communities by using local cloud services. Their context is similar to ours though they assume that the social mechanisms like trust in a small community do not require additional mechanisms such as incentives. The Cloud@Home ${ }^{5}[7]$ project has similar goals to harvest in resources from the community for meeting peaks in resource demands. The system is well described in terms of design and motivation, but a deployed systems seems not yet to be available. The Clouds@ home ${ }^{6}[8]$ project focuses on providing guaranteed performance and ensuring quality of service (QoS) even when using volatile volunteered resources connected by Internet. The authors focus on voluntary computing systems, but do not consider the particular context of community networks. The P2PCS ${ }^{7}[9]$ project has built an initial prototype implementation of a decentralized Peer-to-Peer cloud system. It uses Java JRMI technology and builds an IaaS system that provides very basic support for creating and managing VMs as a slice. It manages slice information in a decentralized manner using gossip protocols. The system is not completely implemented and integrated.

From the review of the related work it can be seen that the socio-technical challenges that we find in community networks are not addressed comprehensively by the related work, and systems are usually not deployed. In the cloud system we propose we put emphasis on the feasibility of deployment and aim that our cloud system is really used. The cloud system proposed in our work should become part of a production system to be used in real community networks.

\section{CONCLUSION}

Community clouds are motivated by the additional value they would bring to community networks. Through community clouds, a vast amount of applications could be deployed in community clouds, boosting the usage and spread of the community network model as ICT infrastructure for society.

This paper presents as approach to bootstrap community clouds a strategy based on deploying on all cloud nodes the Guifi-Community-Distribution (GCODIS), a Linux distribution containing common services and applications, without requiring any specific IaaS platform. A conciously selected

\footnotetext{
${ }^{5} \mathrm{http}: / /$ cloudathome.unime.it

${ }^{6} \mathrm{http}: / /$ clouds.gforge.inria.fr

${ }^{7}$ https://code.google.com/p/cloudsystem
}

combination of design aspects of the approach is presented which should achieve the uptake of the cloud by the community, leading to sustainable community clouds.

The deployment of cloud hosts in Guifi.net is on-going and important steps are already in place to assure that this process is unreversable. Various king of images as ISO or VDI to run pre-installed cloud software has been released. A software repository is created to install, update and upgrade the packages (interactively or unattended), to form the GuifiCommunity-Distro software distribution.

We are now at a point where the feedback from end user participation and user experience is needed to further shape the development of the community cloud components such that this cloud within community networks will ultimately be used as alternative to the commercial clouds in the open Internet.

\section{ACKNOWLEDGMENT}

This work was supported by the European Community Framework Programme 7 FIRE Initiative projects Community Networks Testbed for the Future Internet (CONFINE), FP7-288535, and CLOMMUNITY, FP7-317879. Support is also provided by the Universitat Politcnica de Catalunya BarcelonaTECH and the Spanish Government through the Delfin project TIN2010-20140-C03-01.

\section{REFERENCES}

[1] A. Marinos and G. Briscoe, "Community Cloud Computing," Computing, vol. 5931, no. December, p. 11, Jul. 2009.

[2] M. Bina and G. Giaglis, "Unwired Collective Action: Motivations of Wireless Community Participants," in 2006 International Conference on Mobile Business. IEEE, Dec. 2006.

[3] F. A. Elianos, G. Plakia, P. A. Frangoudis, and G. C. Polyzos, "Structure and evolution of a large-scale Wireless Community Network," in 2009 IEEE International Symposium on a World of Wireless, Mobile and Multimedia Networks \& Workshops. IEEE, Jun. 2009.

[4] K. Bernsmed, M. G. Jaatun, P. H. Meland, and A. Undheim, "Thunder in the Clouds: Security challenges and solutions for federated Clouds," in 4th IEEE International Conference on Cloud Computing Technology and Science Proceedings. IEEE, Dec. 2012, pp. 113-120.

[5] e. a. Braem Bart, "A case for research with and on community networks," ACM SIGCOMM Computer Communication Review, vol. 43, no. 3, pp. 68-73, Jul. 2013.

[6] M. K. Skadsem, R. Karlsen, G. Blair, and K. Mitchell, "Community Cloud - Cloud Computing for the Community," in Proceedings of the 1st International Conference on Cloud Computing and Services Science. Setubal, Portugal: SciTePress, 2011, pp. 418-423.

[7] S. Distefano and A. Puliafito, "Cloud@Home: Toward a Volunteer Cloud," IT Professional, vol. 14, no. 1, pp. 27-31, Jan. 2012.

[8] S. Yi, E. Jeannot, D. Kondo, and D. P. Anderson, "Towards Real-Time, Volunteer Distributed Computing," in 2011 11th IEEE/ACM International Symposium on Cluster, Cloud and Grid Computing. IEEE, May 2011, pp. 154-163.

[9] O. Babaoglu, M. Marzolla, and M. Tamburini, "Design and implementation of a P2P Cloud system," in Proceedings of the 27th Annual ACM Symposium on Applied Computing - SAC '12, New York, USA, Mar. 2012, pp. 412-417. 